\title{
Strengthening BHP's closure framework: a strategy to realise enduring value
}

\author{
J Heyes BHP, Australia \\ T Cooper BHP, Australia
}

\begin{abstract}
BHP developed its original corporate closure framework in 2004, with an emphasis on standardising closure plan content and cost estimation. Since then, the framework has evolved with the release of 'Our Requirements for Closure' (OR Closure) in 2018, including the need for the development of optimised closure outcomes that result in enduring environmental and social benefits.
\end{abstract}

OR Closure considers risks and opportunities to shareholder value and the communities and environment in which we operate. It drives toward optimised closure outcomes for our sites by balancing our values, obligations, safety, costs, and the expectations of external stakeholders.

The OR purposefully shifts the development of 'closure plans' to 'closure management plans' to reflect the dynamic and integrated nature of successful closure planning from the earliest stages of exploration through to closure execution and beyond.

This paper presents the revised closure framework, and how it incorporates the vision for the future state of closure planning within BHP, in its ongoing contribution to global development and its aspiration to leave a sustainable and positive legacy.

Keywords: optimised, legacy, benefits, vision

\section{Introduction}

The original framework for closing BHP mine sites was developed in 2004 to improve consistency in the content of closure plans and cost estimation across BHP. A key driver of the early closure standard was compliance with Sarbanes-Oxley requirements (United States of America 2002), and hence, there was an initial focus on clear processes to enable the development of best estimates for the generation of closure provisions. In addition, there has been a shift in the social acceptability of the historical industry practice of post-closure in-perpetuity management; specifically the misalignment of this with the principles of sustainable outcomes for intergenerational equity. The closure standard was part of a suite of health, safety, environment and community (HSEC) management standards. Iterations over the next 10 years saw the standard shift from being mainly a HSEC consideration to a broader holistic planning matter when it became a component of the 'Our Requirements - Corporate Alignment Planning' (OR CAP) standard; OR CAP being the mandatory, fundamental company-wide planning process across all time horizons. The OR CAP process aims to maximise value by understanding strategic growth options, then focuses on medium- and short-term plans to deliver against these strategic objectives.

The management of impacts and risks associated with mining and petroleum extraction within BHP is through a set of corporate-level documents known as 'Our Requirements' (OR). OR details and defines requirements for business planning, risk management, and the minimum expectations of key process areas to protect and create value for BHP as well as maintaining our social licence to operate. OR also serves as an assurance and audit protocol against which the performance of operations, business units, and supporting corporate functions can be assessed. Today, the minimum requirements for closure management planning are set out in 'Our Requirements for Closure' (OR Closure), which is a stand-alone document governed by the Resource Engineering Centre of Excellence (RE CoE). Importantly, it retains a 
strong link with OR CAP as well as other standard corporate processes, such as the risk management standard, capital projects and the investment process.

Stakeholder engagement was integral in the re-development of OR Closure. BHP's Forum on Corporate Responsibility (FCR) was engaged to discuss the concerns, needs and perceptions of external stakeholders with respect to closure. The outcome of these discussions was the creation of guiding principles for closure, which set the strategic direction for the content of OR Closure. BHP's closure objective is to deliver optimised closure outcomes for our sites. Importantly, OR Closure applies to anyone within BHP who is involved in managing, planning or executing exploration, projects, operational or closure activities for all sites (projects, operations, producing fields, operations in care and maintenance, and closed sites).

\section{What is meant by an 'optimised closure outcome'}

Optimised closure outcomes are those that balance values, obligations, safety, costs and the expectations of external stakeholders to enable one or a combination of divestment, relinquishment, ongoing management, or use of the site for an alternative land use. Achieving optimised closure outcomes will support long-term shareholder value and BHP's license to operate. It will also support BHP and industry in its ongoing contribution to global development and its aspiration to leave a sustainable and positive legacy.

The United Nations Sustainable Development Goal 8 (United Nations 2015) states that, "Sustainable economic growth will require societies to create the conditions that allow people to have quality jobs that stimulate the economy while not harming the environment."

The application of this goal in a post-mining scenario presents a challenge, when high employment opportunities reduce drastically, both from the employment offered by mining directly and the associated supporting businesses. However, subsequent sub-goals provide an insight into how a local economy post-mining could be developed (United Nations 2015):

"8.2 Achieve higher levels of economic productivity through diversification, technological upgrading and innovation, including through a focus on high-value added and labour-intensive sectors."

"8.3 Promote development-oriented policies that support productive activities, decent job creation, entrepreneurship, creativity and innovation, and encourage the formalization and growth of micro-, small- and medium-sized enterprises, including through access to financial services."

"8.9 By 2030, devise and implement policies to promote sustainable tourism that creates jobs and promotes local culture and products."

Heyes et al. (2018) discussed how, in the mining industry, closure objectives committed to closure plans submitted as part of project approvals typically default to the return of native vegetation or to the pre-mining land use. This occurs due to several reasons, such as time pressures to obtain the approval, a lack of long-term data or lack of specific detailed consultation on closure alternatives.

This behaviour can lead to opportunities to deliver greater long-term value being missed. For example, all mine sites have well established infrastructure and utilities during the operational stage, such as power, water and transport networks and in some cases thriving communities. Typically, these facilities are removed at closure, eliminating the development of future economic opportunities.

This paradigm was contested by Murphy \& Heyes (2016) when they highlighted that mining companies had an opportunity to utilise closed mine sites for alternate uses that could be aligned with regional development strategies with the potential to provide inter-generational scale benefits to host communities.

An advantage of developing an alternate land use as part of an optimised closure outcome is that, in many cases, it will increase the likelihood of there being an active onsite presence and therefore reduce unauthorised access or provide for 'safe' access. 
The recently released International Council on Mining and Metals (2019) Integrated Mine Closure Good Practice Guide reinforces the concept of optimised closure outcomes, where it discusses the need to look for beneficial uses as part of post-closure land-use planning.

In addition to these economic objectives, public safety must also be considered. In a little over 12 months in Western Australia, there were three public fatalities on mine pit lakes at Red Hill, Pardoo and Lake Stockton. Whilst one of these tragic accidents occurred on a mine lake where public access was encouraged, two of them occurred on unmanned, private properties. Parshley \& Macallum (2016) applied the term 'attractive nuisances' to closed mine sites, highlighting that whilst professionals from the mining industry are well versed in the dangers that mining presents, both in operations and into closure, the public do not have the same level of risk appreciation. Whilst some risks may be more obvious such as rockfalls or highwalls, less visible risks associated with pit lakes, such as poor water quality or submerged hazards may also exist. "If these conditions present an inviting opportunity, they could be considered an attractive nuisance that invites trespass and puts the trespasser at risk" (Parshley \& Macallum 2016).

Heyes et al. (2018) observed that, "Whilst current social license to operate philosophies encourage corporate responsibility, there is limited financial incentive for mining companies to drive approval for an alternative land use and extend their responsibility beyond relinquishment, which they may view as more of a long-term host-government responsibility". However, as Blackrock CEO Laurence Fink (2018) noted in his recent letter "Society is demanding that companies, both public and private, serve a social purpose. To prosper over time, every company must not only deliver financial performance, but also show how it makes a positive contribution to society."

The development of optimised closure outcomes that are accepted and embraced by stakeholders will be an important aspect for mining companies to demonstrate their commitment to social value.

\section{Closure management process}

BHP's delivery of optimised closure outcomes is achieved by following the closure management process, contained within OR Closure (Figure 1).

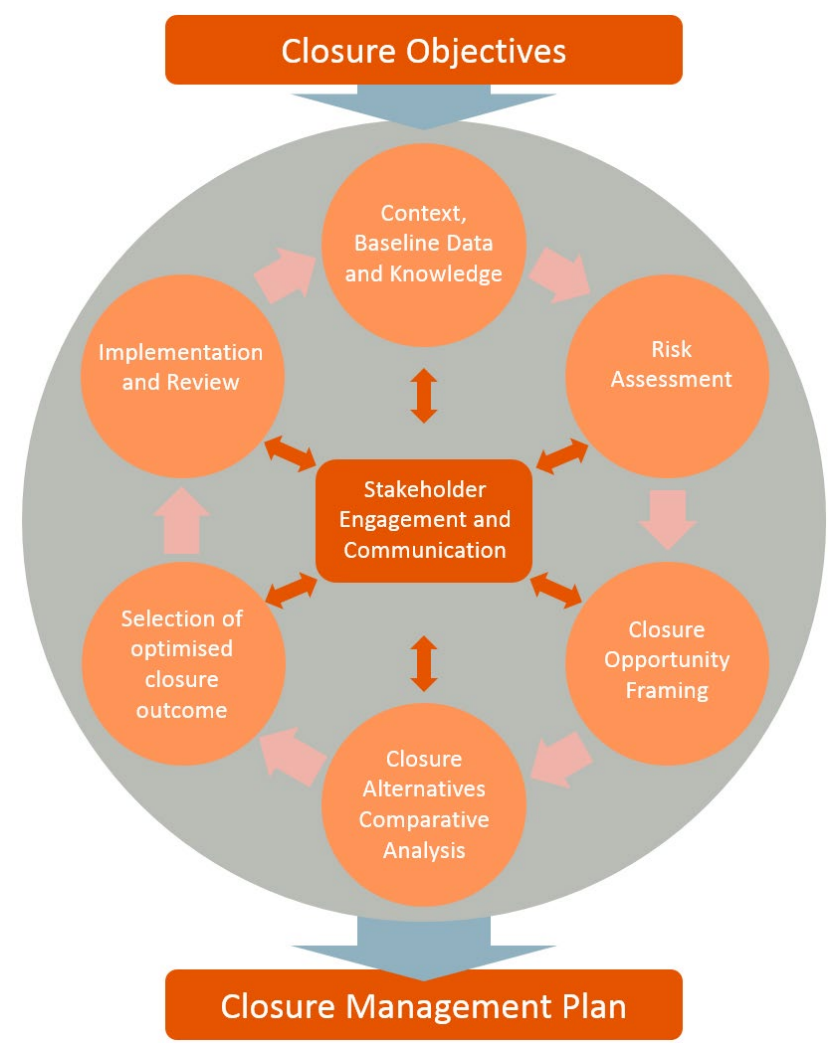

Figure 1 BHP's 'Our Requirements for Closure' - closure management process 
The iterative steps of the closure management process are designed to enable development of closure management plans that meet closure objectives. Central to the closure management process is ongoing stakeholder engagement and communication.

Kalma (2018) discussed the need for stakeholder engagement to start at the exploration stage and continue throughout operations into closure, noting that, "Social performance requires cooperation between all parts of the business and for company values to be aligned" and that, "attitudes and behaviours of company representatives during early engagement can impact company reputation."

The output of the closure management process is a comprehensive closure management plan. This term has been developed to emphasise that closure planning is not a one-off, static activity. Closure must be managed in such a way that it is integrated in existing business processes. Crucially, strong links between OR Closure and OR CAP have been maintained, with closure management plans being an input and an output of lifeof-asset plans, with shorter term actions being carried forward into five-year plans. This ensures that there is consistency between long- and short-term plans and budgets.

Each element of the closure management process will be described in the following sections.

\subsection{Context and baseline knowledge}

The original collection and ongoing update of context and baseline knowledge of the site is required to make sure that the closure management plan continues to meet the expectations of all stakeholders, as well as including changing environmental and social context, such as climate change, community demographics, third party activity, water demand and supply, and changing mine plans.

An example of the importance of this is that of supporting local communities. Throughout the life of a mine, the community may experience ebbs and flows in the supply and demand for accommodation and associated supporting infrastructure (e.g. schools). Early in a mine life, demand will be high, with supply gradually meeting demand, followed by decreases associated with lows in the commodity cycle. Demand will be then be high, with expansions or new satellite mines, ultimately followed by a decrease into closure. Is it appropriate for a closure plan to be based on supporting the original community structure, peak cycle or somewhere in between? With this in mind, OR Closure requires a community exit strategy to be developed and updated as part of the closure management plan throughout the life-of-mine.

Reflecting the cyclical nature of the closure management process, information gaps must be identified and addressed throughout the operational life. The imperative here is that sufficient baseline data covering aspects from asset infrastructure, environment and social values is available and of a quality to provide solid inputs to the risk assessment. Baseline datasets that capture spatial and temporal variability in the asset or facility considered for closure is a critical step contributing to stakeholder confidence in the closure outcome.

\subsection{Risk assessment}

Risk assessments are a widely used tool in the mining industry, however, many tools focus on tactical or operational risk and do not well serve the lengthy time frames associated with closure. In recognition of this, OR Closure requires the assessment of specific closure risks, including the assessment of the risk of unplanned closure, pre-closure risks (before or during closure execution) and post-closure risks (after closure execution) of the site, risks associated with social and environmental, direct, cumulative, and indirect impacts (such as climate change), and potential changes to regulations.

BHP's standard risk assessment process is used for closure risk assessments to ensure consistency. It contains specific requirements for the assessment, control, monitoring, and reporting of risks. Management of risks related to closure frequently requires effective controls to be in place during operations, with responsible control owners holding senior operational positions. One example of this is the risk of acid and metalliferous drainage (AMD), for which a specific group-wide technical standard is in place. The technical standard follows a plan, do, check, act approach, with an overarching strategy that prevention (source control) of AMD, rather than treatment in perpetuity, is the preferred lower risk approach. 


\subsection{Closure opportunity framing}

Opportunity framing is a routine BHP process governed by the investment evaluation team and follows a consistent methodology for the identification of opportunities and risks. Estimates of value and evaluating investments is completed through an objective assessment of the risks and merits of each investment and supports informed decision-making. Closure is considered in the same manner as any other investment.

Opportunity framing uses a structured approach to the divergent thinking that is required to generate a range of credible value-creating investment alternatives in the early part of the conceptual study phase of a project. It includes independently facilitated workshops to synthesise the knowledge and experience of the group, overcome motivational and cognitive bias, and assist with generating a complete range of reasonable alternatives.

\subsection{Closure alternative comparative analysis}

A comparative analysis is a process used to compare and contrast the range of closure options from 'do nothing' through to full closure (often conditioned in the approvals process as the return to prior land use) including options between these endpoints to identify the optimised closure outcome for mining or petroleum activities. Comparative analysis is technical framework that is able to accommodate both science-based and values-based objectives, and deal with uncertainty in information. The output of the process is a ranking of options in their relative order of merit based on evaluation against criterion and the risks associated with each criterion, to arrive at a recommendation of the overall merit of an option against all requirements.

The BHP comparative analysis process has four key steps each supported by stakeholder engagement as described in Figure 2.

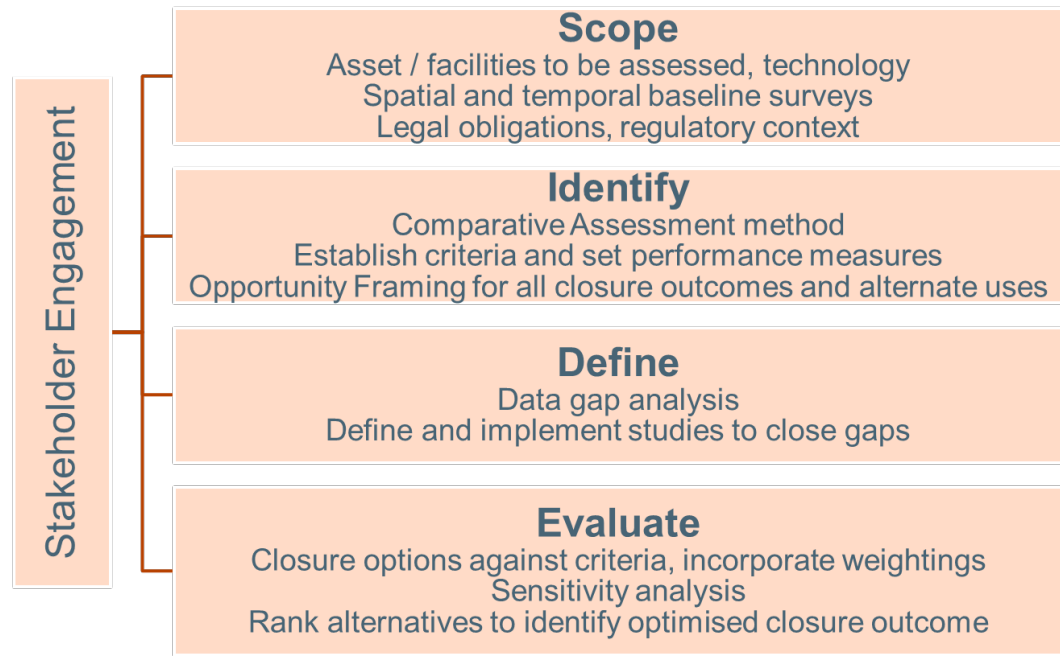

Figure 2 Comparative analysis process

'Scoping' comprises an understanding of the asset/facilities to be assessed, forming a multi-disciplinary team to be involved, reviewing the perspectives of internal and external stakeholders likely to be affected by the closure activity and a review of current legal obligations and/or the regulatory context for a site.

'Identification' includes establishing evaluation criteria developed to allow the comprehensive comparative ranking of the closure options/alternatives. The evaluation criteria generally address aspects ranging from HSE, cultural heritage, social value, and finance. Objectives are measurable levels of performance required for each evaluation criteria to ensure that the impacts and risks associated with management of a closure option/alternative will be of an acceptable level. Measurement criteria define how performance is measured and determine whether the objectives have been (or are likely to be) achieved. 
The identification phase should also include opportunity framing workshops to identify all closure outcomes (from 'do nothing' as base-case, to full closure), compilation of alternate land uses possible for a site, and then short-listing of those options and alternate uses. A key step in this phase is a determination on the comparative analysis method to be used; complex closure options with increased public scrutiny will require robust, quantitative assessment approaches (e.g. multi-criteria decision analysis [MCDA]; discussed further below).

'Definition' should focus on understanding any key data gaps associated with the short-list of closure options and alternate uses followed by the implementation of studies to close data gaps. These should align with the evaluation criteria established in the previous step.

'Evaluation' involves assessment of closure options against criteria to identify the optimised closure outcome. The data inputs should be sufficiently comprehensive to enable assessment on the practicability of each of the closure options considered, and allow for an evaluation where selection of the optimised outcomes are based on scientific principles of independence and replication. The evaluation criteria are scored with ratings and a weighting applied based on their relative importance, which can be adjusted to understand how alternatives perform in different scenarios. Weightings also allow sensitivity analysis to be used to test the robustness of the results and assess identification of preferred options when different perspectives are applied. Those that consistently rank highest can be subject to further studies or assessments, prior to engagement with stakeholders and recommending a decision for management approval/endorsement.

Selection of the evaluation method will vary depending on the complexity of the closure project, but one such approach that has been used in BHP successfully is MCDA. MCDA involves the development of a matrix where each column describes the closure options (from full closure activity through to the 'do nothing' option, and combinations thereof) and each row describes the performance of the options against each weighted valuation criterion (as described above). The process involves the scoring of each option for each criterion, and then combines the product of the weight and score to yield an overall sum, which is then ranked, for each option. The outcome is an overall order of options, from the most preferred to the least preferred option. MCDA has many advantages over purely qualitative approaches or those based on professional judgement. It is transparent and allows one to easily look 'under the hood' to understand how criteria have been weighted and performance measures scored, leading to increased confidence in the result. It provides an audit trail of the considerations, data inputs and justifications supporting scores within performance criteria. It removes inherent biases of those undertaking the closure planning and design, supports the stakeholder engagement process, and demonstrates to stakeholders that all options have been considered and rationales for the preferred options have been identified. Sensitivity analyses allow examination of closure options from different perspectives and allow comparison of the output with and without implementation cost to avoid critique that only low-cost options are preferred. Importantly, the approach can incorporate both quantitative and qualitative judgements.

'Stakeholder engagement' is integrated into each of the steps above and is key to the success of the comparative analysis outcome. Ideally, stakeholders (internal and external) are engaged at each step of the process from scoping the asset or facility and providing input to the baseline data gathering/review step; identification by input to the development of the evaluation criteria and performance measures; definition by review of technical studies to close gaps; and finally the evaluation process itself.

The overall objective of the comparative analysis is to facilitate stakeholder engagement in the process to enable understanding and acceptance of the process to support the outcome (i.e. identification of the optimised closure outcome).

\subsection{Selection of optimised closure outcome}

An output of the comparative analysis is the selection of the preferred optimised closure outcome. A key step here is further stakeholder engagement to discuss the comparative analysis process including discussion to agree on the optimised closure outcome. Allowance in the closure schedule is made for any adjustments and re-evaluation, if necessary, to align with stakeholder expectations further supporting acceptance of the 
outcome. Where opportunities have been identified, but there is insufficient technical data to support their inclusion in the 'base' closure plan, a work plan is developed that will close the knowledge gaps before the next iteration of the plan. It is important to note that the optimised closure plan will likely differ to the outcomes contained within external closure plans required by regulation in the absence of adaptive processes whereby new information that may lead to better closure outcomes can be incorporated into approved closure plans.

\subsection{Implementation and review}

The final step of the closure management process is the definition and implementation of detailed work plans. The plans need to describe the pre-closure activities needed to achieve the optimised closure outcome and completion criteria. These include risk management, technical work, design criteria, monitoring, performance indicators, and scheduling of activities. It is critical to undertake this work throughout the mining lifecycle to ensure that the knowledge base is sufficiently mature, before closure projects enter into a pre-feasibility study phase. As knowledge gaps are closed, opportunities identified in earlier stages may become the 'optimised closure outcome' during future plan revisions.

\section{Conclusion}

For greenfield developments, leaving behind a negative mining legacy that requires in-perpetuity treatment and management is no longer socially acceptable and the mining industry must push toward sustainable outcomes for closure and be aligned to the principles of intergenerational equity.

OR Closure has been designed to develop 'optimised closure outcomes' that meet this challenge. The roll out of OR Closure across BHP has emphasised that execution of the closure management plan does not just apply to the execution of closure projects; it relates to the ongoing implementation of the closure management plan throughout the operational life. To reflect this, the term 'closure plan' has been replaced with 'closure management plan'.

Plans to progressively reduce closure obligations, the current and future disturbance area, and redundant infrastructure during operations, must be defined, along with the key activities and timing to implement the closure project, and be documented in the closure management plan.

Post-closure activities to achieve the optimised closure outcome, including risk management, technical work, monitoring, management and operational activities, and schedule must also be included in the closure management plan.

The integration of closure planning into key business process at BHP has evolved over the past 15 years; from a stand-alone document in the HSE management system, moving to OR CAP and now going full circle back to an independent OR but retaining critical links to OR CAP. The OR CAP process is fundamental to creating alignment across BHP; it guides the development of plans, targets and budgets to help decide on allocation of capital and resources. The OR CAP process starts with planning for the long-term (life-of-asset includes closure timeframes) to maximise value by understanding our strategic options for growth, then focuses on medium- and short-term plans to deliver against these strategic objectives. The inclusion of closure requirements as mandatory inputs and outputs in the planning process is a core concept that enables the optimised closure outcome to be realised and sustains a social licence to operate.

As Laurance (2006) wisely pointed out, "The excitement and fanfare that surrounds the opening of a new mine is never present when it finally closes". Therefore, we must generate the excitement by integrating closure into both the day-to-day management of mines and the long-term plans, inspiring collaborative and integrated action that will result in optimised closure outcomes.

\section{Acknowledgement}

The authors thank Evelyn Bingham for her pioneering work developing the original BHP closure standard. 


\section{References}

Fink, L 2018, 'BlackRock's message: contribute to society, or risk losing our support', New York Times, viewed 12 March, 2018, https://www.nytimes.com/2018/01/15/business/dealbook/blackrock-laurence-fink-letter.html

Heyes, J, Murphy, D, Foster, S \& White, S 2018, 'What comes after mining? How regulatory frameworks can enable creativity', in C Drebenstedt, F von Bismarck, AB Fourie \& M Tibbett (eds), Proceedings of the 12th International Conference on Mine Closure, Technical University Bergakademie Freiberg, Freiberg, pp. 173-182.

International Council on Mining and Metals 2019, Integrated Mine Closure: Good Practice Guide, London, https://guidance.miningwithprinciples.com/integrated-mine-closure-good-practice-guide

Kalma, T 2018, 'Exploration and early engagement', Aus/MM Bulletin, December 2018.

Laurence, D 2006, 'Optimisation of the mine closure process', Journal of Cleaner Production, vol. 14, pp. 285-298.

Murphy, D \& Heyes, J 2016, 'Mine closure - are we using the right drivers?', AusIMM Bulletin, October 2016.

Parshley, JV \& Macallum, CS 2016, 'Attractive nuisances and wicked solutions', in AB Fourie \& M Tibbett (eds), Proceedings of the 11th International Conference on Mine Closure, Australian Centre for Geomechanics, Perth, pp. 229-240.

United Nations 2015, Sustainable Development Goals, New York.

United States of America 2002, Sarbanes-Oxley Act 2002. 